\title{
AN INVERSE PROBLEM FOR BIHARMONIC EQUATION
}

\author{
A.G. RAMM \\ Department of Mathematics \\ Cardwell Hall \\ Kansas State University \\ Manhattan, KS 66506 \\ (Received March 18, 1987)
}

ABSTRACT. An inhomogeneity immersed in a medium is found from the measurements of the elastic field on the surface of the medium.

KEY WORDS AND PHRASES. Biharmonic equation, Cauchy problem, Inverse problem, elastic problem in inhomogeneous medium.

1980 AMS SUBJECT CLASSIFICATION CODE. 73D50, 73C40.

1. INTRODUCTION.

Consider the biharmonic equation

$$
\Delta^{2} u=0 \text { in } R_{-}^{2}=\{x, y: y<0\}
$$

and the boundary conditions

$$
\begin{gathered}
u(x, 0)=u_{0}(x), \quad u_{y}(x, 0)=u_{1}(x), \\
\Delta u(x, 0)=u_{2}(x), \quad \frac{\partial \Delta u(x, 0)}{\partial y}=u_{3}(x) .
\end{gathered}
$$

Given the data $(1.2)$ one wishes to find a surface $\Gamma \subset R_{-}^{2}$ such that

$$
\mathbf{u}=0 \text { on } \mathrm{r} \text {. }
$$

One can use some other boundary conditions on the unknown $\Gamma$, such as $u_{N}=0$, for example. Here $\mathrm{N}$ is the direction of the normal to $\mathrm{r}$.

The basic idea of this paper is similar to the one in [1]. Namely, we measure the solution $u$ to an elliptic equation and the normal derivatives or other combinations of the derivatives of the solution on a certain manifold (on the line $y=0$ in our particular case) and we wish to recover an unknown surface $\Gamma$ (if such a surface exists) on which $u$ vanishes (or certain differential expression applied to $u$ vanishes). The basic fact behind this idea is the uniqueness of the solution to the Cauchy problem for elliptic equations: if one knows on a manifold the Cauchy data for an elliptic equation then the solution is uniquely determined. For elliptic equation (1.1) of the fourth order there are four Cauchy data which we have chosen in (1.2). 
The objective of this paper is to give a method for finding the unknown $\Gamma$ on which condition (1.3) holds from the knowledge of the data (1.2). The method works for other than (3) boundary conditions and for other than (1.1) elliptic equations.

Finally we wish to note that the problems of the type we discuss can be of interest in applications. For example in the plane static problems of elasticity the deformation vector can be expressed in terms of the scalar solution $x$ of the biharmonic equation (this solution is called the deformation function). The boundary conditions on the surface $\Gamma$ of the inhomogeneity immersed in the elastic medium can be expressed in the form similar to (1.3): certain differential expressions involving $\chi$ vanish on $\Gamma$. We wish to find $r$ (for example, the boundary of a crack) from the measurements of $u$ on a certain surface.

2. A METHOD FOR SOLVING THE PROBLEM.

A. We wish to show that there is a solution to equation (1.1) which is of the form

$$
\begin{aligned}
u(x, y) & =\int d \alpha\left\{\left[A_{0}(\alpha)+x A_{1}(\alpha)+y A_{2}(\alpha)\right] \exp (i \alpha x+\alpha y)\right. \\
& \left.+\left[B_{0}(\alpha)+x B_{1}(\alpha)+y B_{2}(\alpha)\right] \exp (i \alpha x-\alpha y)\right\}
\end{aligned}
$$

where $\int:=\int_{-\infty}^{\infty}$, and $A_{j}(\alpha), B_{j}(\alpha)$ are to be chosen so that the boundary conditions (2.1) are satisfied. For arbitrary $A_{j}(\alpha), B_{j}(\alpha)$ decaying at infinity sufficiently fast, so that the integral in (2.1) can be differentiated four times in $x$ and $y$ under the sign of the integral, the function (2.1) solves equation (1.1). For the function (2.1) to satisfy conditions ( 1.2$)$, the following equations should hold:

$$
\begin{aligned}
& \tilde{u}_{0}=A_{0}(\alpha)+B_{0}(\alpha)+i A_{1}^{\prime}+i B_{1}^{\prime} \\
& \tilde{u}_{1}=A_{2}(\alpha)+B_{2}(\alpha)+\alpha A_{0}(\alpha)-\alpha B_{0}(\alpha)+i\left(\alpha A_{1}\right)^{\prime}-i\left(\alpha B_{1}\right)^{\prime} \\
& \tilde{u}_{2}=2 i \alpha\left(A_{1}(\alpha)+B_{1}(\alpha)\right)+2 \alpha\left(A_{2}(\alpha)-B_{2}(\alpha)\right) \\
& \tilde{u}_{3}=2 i \alpha^{2} A_{1}(\alpha)+2 \alpha^{2} A_{2}(\alpha)-2 i \alpha^{2} B_{1}(\alpha)+2 \alpha^{2} B_{2}(\alpha) .
\end{aligned}
$$

Here $A^{\prime}=d A / d \alpha, \tilde{\mathbf{f}}:=(2 \pi)^{-1} \int f(x) \exp (-i \alpha x) d x$ and the Fourier transform is taken in the sense of distributions. We have 6 functions $A_{j}, B_{j}, j=0,1,2$, to satisfy four equations (2.2) - (2.5). Therefore two additional conditions can be imposed. If, for example, we choose these additional conditions in the form

$$
A_{1}=0, \quad B_{1}=0,
$$

then (2.2) $-(2.6)$ is a linear system of six equations for six unknown functions $A_{j}, B_{j}$, $j=0,1,2$, which can be solved explicitly. Indeed, it follows from (2.6) that equations $(2.2)-(2.5)$ can be written as

$$
\begin{aligned}
& \tilde{u}_{0}=A_{0}+B_{0} ; \tilde{u}_{1}=\alpha\left(A_{0}-B_{0}\right)+A_{2}+B_{2} ; \tilde{u}_{2}=2 \alpha\left(A_{2}-B_{2}\right) \\
& \tilde{u}_{3}=2 \alpha^{2}\left(A_{2}+B_{2}\right) .
\end{aligned}
$$


This system has the unique solution:

$$
\begin{aligned}
& A_{2}=\left[(2 \alpha)^{-1} \tilde{u}_{2}+\left(2 \alpha^{2}\right)^{-1} \tilde{u}_{3}\right] / 2, B_{2}=\left[\left(2 \alpha^{2}\right)^{-1} \tilde{u}_{3}-(2 \alpha)^{-1} \tilde{u}_{2}\right] / 2, \\
& A_{0}=\left[\tilde{u}_{0}+\alpha^{-1} \tilde{u}_{1}-\left(2 \alpha^{2}\right)^{-1} \tilde{u}_{3}\right] / 2, B_{0}=\left[\tilde{u}_{0}-\alpha^{-1} \tilde{u}_{1}+\left(2 a^{2}\right)^{-1} \tilde{u}_{3}\right] / 2 .
\end{aligned}
$$

Formulas (2.1), (2.6) and (2.8) give an explicit analytical formal solution to problem (1.1)-(1.2). This solution is smooth in $x$ and $y$ in $R_{-}^{2}$ provided that the data $u_{j}(x)$, $0 \leqq j \leqq 3$, are such that the functions defined by formulas (2.8) decay sufficiently fast as $\alpha \rightarrow \pm \infty$, for example, faster than $\exp (-a|\alpha|)$ with any $a>0$, so that the integral (2.1) converges and can be differentiated under the sign of integral.

B. Having solved problem (1.1) - (1.2), we can find the unknown boundary $\Gamma$ from equation (1.3). Indeed, if the function $u(x, y)$ is found, one can use the equation

$$
u(x, y)=0
$$

as an implicit equation of $\Gamma$. If $y=y(x)$ is an implicit function determined by equation (2.9) then $y=y(x)$ is an equation of a component of $\Gamma$.

This completes the description of the idea of the method for solving the inverse problem formulated in section $I$. The choices of the additional two conditions other than (2.6) are possible.

REMARK 1. This problem is $i 11$ posed because the Cauchy problem for elliptic equations is $i l 1$ posed. Not for any data $u_{j}(x), j=0,1,2,3$, formulas (2.8) define the functions $A_{0}, A_{2}, B_{o}, B_{2}$ such that integral (2.1) makes sense (see Example 2 below).

EXAMPLE 1. Let us take the data such that $A_{0}(\alpha)=1$ if $-1 \leq \alpha \leq 1$ and $A_{0}(\alpha)=0$ otherwise, $A_{1}=B_{1}=A_{2}=B_{2}=B_{0}=0$. Then $u(x, y)=\int_{-1}^{1} d a \exp (i \alpha x+a y)=2(i x+y)^{-1}$ $\sinh (i x+y)$. Equation (2.9) for this $u(x, y)$ is $\sinh (i x+y)=0$. It has solution $y=0$, $x=n \pi, n=0, \pm 1, \pm 2 \ldots$ Thus, there is no curve $\Gamma$ in $R^{2}$ corresponding to the above data.

EXAMPLE 2. Consider $u(x, y)=x^{2}+\left(y+\frac{1}{2}\right)^{2}-\frac{1}{4}$. Clearly $\Delta^{2} u=0, u_{0}(x)=x^{2}, u_{1}(x)=1$, $u_{2}(x)=4, u_{3}(x)=0$. Thus $\tilde{u}_{0}=-\delta^{\prime \prime}(\alpha), \widetilde{u}_{1}=\delta(\alpha), \tilde{u}_{2}=4 \delta(\alpha), \tilde{u}_{3}=0$, where $\delta(\alpha)$ is the delta-function. Since the expression $\alpha^{-1} \delta(\alpha)$ is not well defined, formula (2.8) for the data in this example cannot be used. One can represent the function $u(x, y)=x^{2}$ $+\left(y+\frac{1}{2}\right)^{2}-\frac{1}{4}$ in the form (2.1) if one does not assume that $A_{1}=B_{1}=0$.

\section{REFERENCES}

1. RAMM, A.G., 1986, A geometrical inverse problem, Inverse problems, 2, L19-L21.

2. LANDAU, L. and LIFSHITZ, E., 1970, Theory of elasticity, Pergamon Press, New York. 


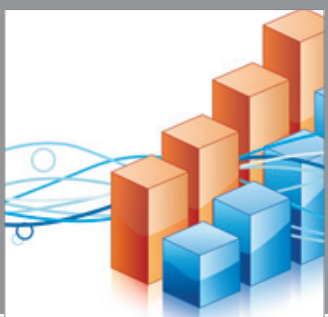

Advances in

Operations Research

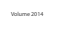

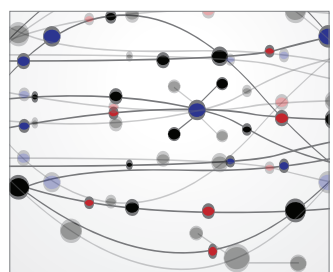

\section{The Scientific} World Journal
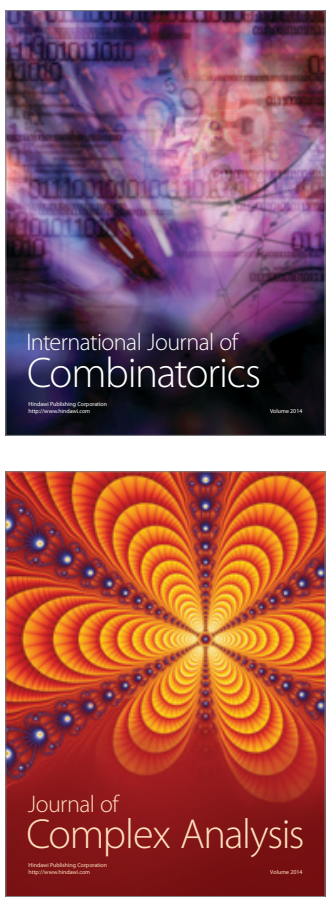

International Journal of

Mathematics and

Mathematical

Sciences
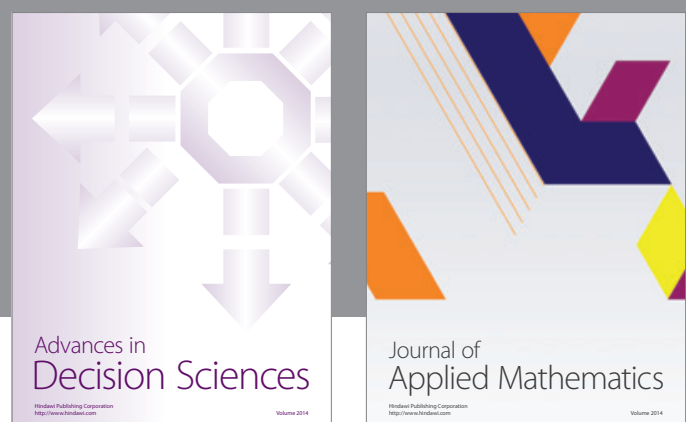

Journal of

Applied Mathematics
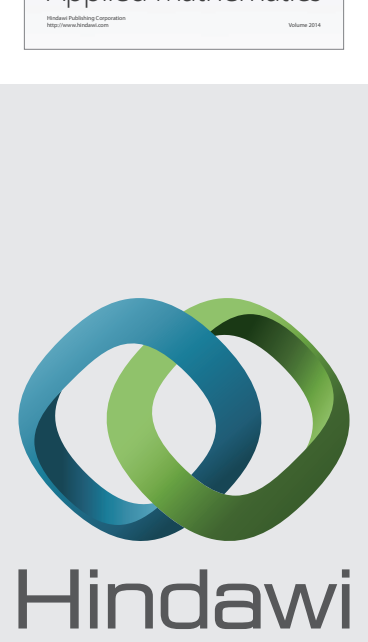

Submit your manuscripts at http://www.hindawi.com
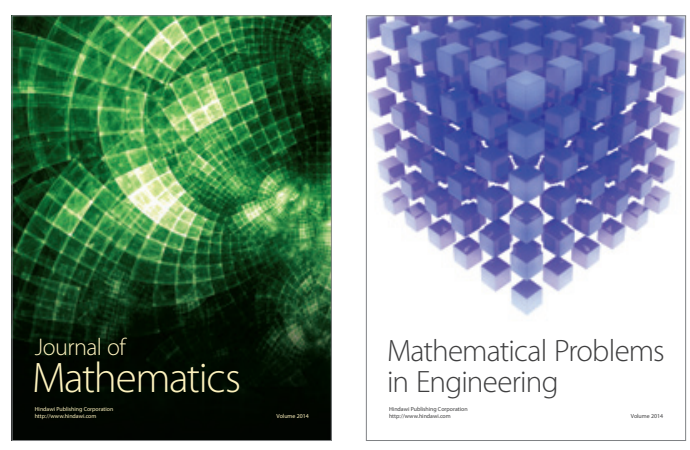

Mathematical Problems in Engineering
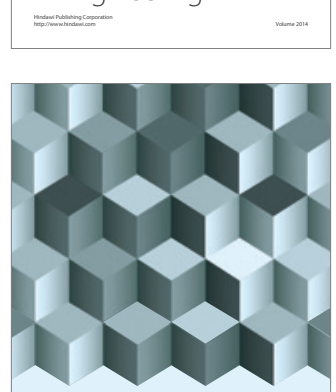

Journal of

Function Spaces
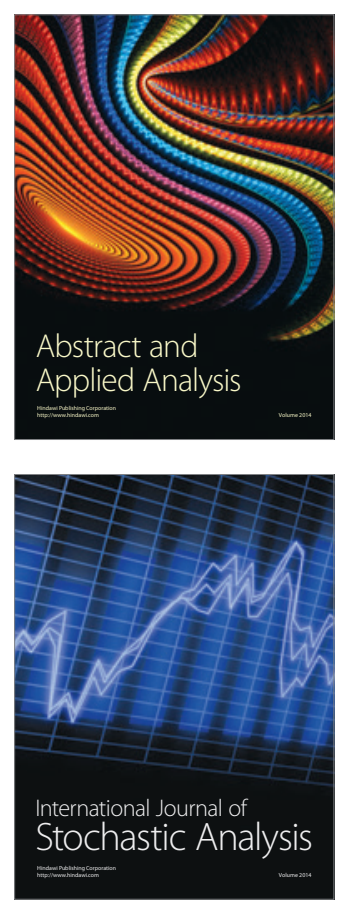

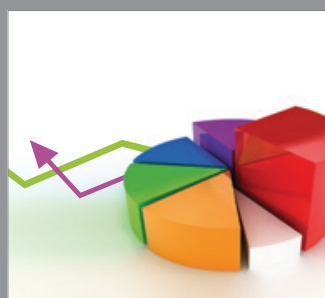

ournal of

Probability and Statistics

Promensencen
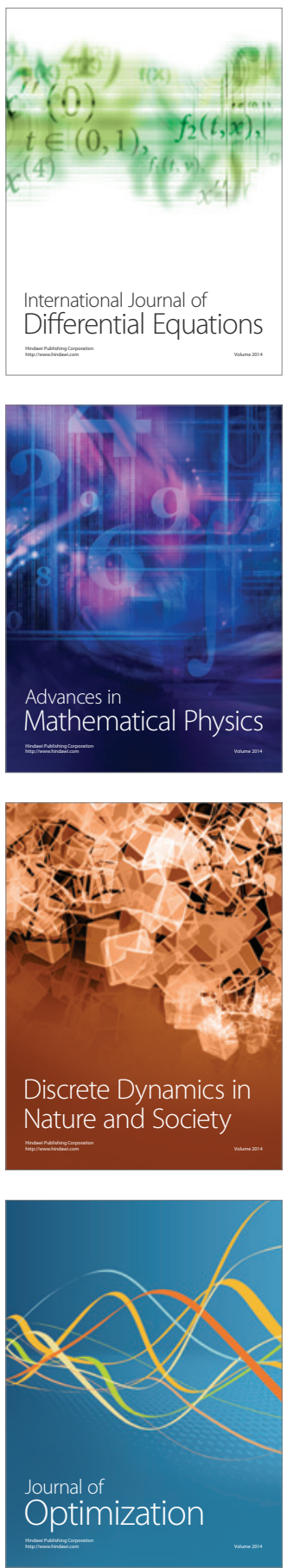